\title{
The Large Magellanic Cloud and the Distance Scale
}

\author{
Alistair R. Walker ${ }^{1}$ \\ Cerro Tololo Inter-American Observatory, National Optical Astronomy Observatory, \\ Casilla 603, La Serena, Chile. \\ awalker@ctio.noao.edu
}

Received — 


\begin{abstract}
The Magellanic Clouds, especially the Large Magellanic Cloud, are places where multiple distance indicators can be compared with each other in a straightforward manner at considerable precision. We here review the distances derived from Cepheids, Red Variables, RR Lyraes, Red Clump Stars and Eclipsing Binaries, and show that the results from these distance indicators generally agree to within their errors, and the distance modulus to the Large Magellanic Cloud appears to be defined to $\pm 3 \%$ with a mean value $(m-M)_{0}=18.48$ mag, corresponding to $49.7 \mathrm{Kpc}$. The utility of the Magellanic Clouds in constructing and testing the distance scale will remain as we move into the era of Gaia.
\end{abstract}

Subject headings: Distance Scale; Magellanic Clouds 


\section{Introduction}

The Magellanic Clouds (MC) are the closest populous galaxies to our own, and as such contain significant numbers of most of the indicators used to make up the distance ladder that transfers geometrically measured distances for nearby stars to the far universe via multiple overlapping steps. Both galaxies are now realized to be complex systems interacting with each other but not perhaps as-yet with the Galaxy (Nidever et al. 2010; Olsen et al. 2011; Besla et al. 2011).

The main structures of the Large Magellanic Cloud (LMC) lie reasonably close to the plane of the sky (Nikolaev et al. 2004), but the Small Magellanic Cloud (SMC) has long been realized (Caldwell \& Coulson 1986) to form an extended structure almost in the line of sight. Although at times a Galaxy-LMC-SMC comparison is useful to test the metallicity dependence of some particular distance indicator, in general the SMC is of rather less utility than the LMC for distance scale work. The approximation that the LMC is sufficiently compact and distant that its contents are all at the same distance from us - and yet close enough that crowding and faintness are usually not issues - is a highly valuable attribute, although when pushing for distance comparisons at the few percent level the effects of sample size and spatial distribution will be important, and corrections for the geometry of the LMC, should be carefully considered. For small samples the possible line of sight variation in distance to any particular star must be taken into account as it may be significantly different from the mean distance, and for unique distance indicators such as SN 1987A the distance determined is of course that for the object itself and to go from that distance to the mean distance of the corresponding galaxy requires an extra step with its own associated error. In some cases the latter may be difficult to determine (Saha et al. 2010; Olsen et al. 2011).

Over the past decade the controversy over the "long versus short" distance scale has 
largely been resolved, with the corresponding values of the Hubble Constant $(\sim 50$ vs. $\sim 100$ respectively), settling to an intermediate value, with rather small errors usually quoted (Freedman \& Madore 2010; Riess et al. 2011). Although the range of LMC distances given by all available indicators in an oft-quoted compendium by Benedict et al. (2002) is large, some of the more notable outliers (e.g. RGB clump stars) are now better understood and more nuanced evaluations have generally been consistent for the distance indicators considered most reliable (Walken 2003; Alves 2005; Clementini 2008), to ten percent or better. Does this mean that the distance scale is now rather a less important topic than when apparently careful studies obtained values for the Hubble Constant that were a factor-of-two different? Today in the era of precision cosmology, as typified by the Wilkenson Microwave Anisotropy Probe (WMAP) results (Komatsu et al. 2011), the Hubble Constant is generally used as a prior in the interpretation of the relations that describe the components that make up the energy-density of the Universe. Knowing the Hubble Constant to a (very) few percent is thus essential (Freedman \& Madore 2010) and so we conclude that the study of distance indicators is still of significant scientific importance.

The improvement of instrumentation over the past decade and a half, chiefly the availability of the Hubble Space Telescope (HST) with its series of imaging cameras (WFPC2, ACS, WFC3), has allowed the Cepheid distance scale to be extended well beyond the Local Group. Additionally, measurements with the Fine Guiding Sensors (FGS) have allowed a direct calibration by parallaxes for 10 Galactic Cepheids (Benedict et al. 2007), and with a geometrical distance to the maser galaxy "NGC 4258" (Macri et al. 2006) available for comparison, the "one step" jump to the Supernovae Ia (SNIa) that are used to probe to great distances would seem to reduce the importance of the MC in distance scale work. But the history of the subject shows systematic errors are invidious; the well known adage that "The Hubble Constant at any given time has always been known to 10 
percent, despite having changed over that period by a factor of 10 " should not be forgotten. For these reasons the importance of the MC, and especially the LMC remains intact, providing a sanity check on the validity of the lower rungs of the distance scale ladder. The availability of large telescopes with relatively wide-field imagers (e.g. Subaru, Magellan, LBT) means that such comparisons are not restricted to the MC, with the more luminous distance indicators able to be compared for instance in M31 and M33.

The discussion here is not meant to be inclusive of all distance indicators that have ever been suggested, and specifically excludes those that at the present time have insecure calibration or large errors. The meeting associated with this review had as its focus The Gaia Perspective and thus the distance indicators chosen here: Cepheids, Red Variables, RR Lyraes, Red Clump Stars, Eclipsing binaries - all have their zero-point calibrations depending on fundamental geometric methods. These calibrations will be greatly improved by Gaia, and some other distance indicators can be expected to join this list. This selection does of course introduce some bias of its own, which the reader is well advised to bear in mind (Schaefer 2008). Unless stated otherwise the distances tabulated are those determined by the authors of the papers quoted. The recent comprehensive review of pulsating variable stars in the MC by Clementini (2008) is highly recommended for a discussion complementary to that here; other important recent discussions are those of Tammann et al. (2008); Freedman \& Madore (2010) both of which contain copious references to earlier work.

\section{Cepheids}

The zero-point of the Cepheid period-luminosity (PL) relation (Leavitt Law) is now best determined from the parallax measurements for 10 Cepheids determined directly with HST FGS (Benedict et al. 2002, 2007). The results can be combined with data for 
Cepheids in clusters and associations (Feast \& Walker 1987; Turner \& Burke 2002) or with pulsation parallaxes (Baade-Wesselink method) e.g. Fouqué et al. (2007) to form accurate PL relations covering a wide period range (Turner 2010). The average error in the individual HST Cepheid parallaxes is $8 \%$, it is noteworthy that these errors are typically a factor 10 smaller than those measured for 220 Galactic Cepheids by Hipparcos, as discussed by Feast \& Catchpole (1997). Benedict et al. (2007) compare their fitted galactic PL relations to those derived from several studies and find that the slopes of the $K$ and Wesenheit $W(V, I)$ band passes are identical in the Galaxy and the LMC to within the errors, and derive a $W(V, I)$ distance modulus $(m-M)_{0}$ of $18.50 \pm 0.03$, with no correction for any metallicity effect on the Cepheid absolute magnitudes. An analysis combining the two data sets (Hipparcos, HST) by van Leeuwen et al. (2007) produces very similar results: LMC moduli of $18.52 \pm 0.03\left(W_{V I}\right), 18.47 \pm 0.03$ ( $K$ mag PL relation), and $18.45 \pm 0.04$ ( $J, K$ mag period-luminosity-color relation), again with no correction for possible metallicity effects. The effectiveness in using directly measured Cepheid parallaxes is obvious, and the improvement that will come with Gaia is enormous, with almost half the 20.000 estimated Cepheids in our Galaxy being accessible (Windmark et al. 2011).

The concerns when transferring a Cepheid PL relation from the Galaxy to the LMC and beyond mostly are regarding the effect of metal abundance on the slopes (Gieren et al. 2005; Fouqué et al. 2007; Madore \& Freedman 2009) and zero-points (Bono et al. 2008; Kudritzki et al. 2008; Scowcroft et al. 2009; Storm et al. 2011a; Freedman \& Madore 2011) of the PL relation. Careful choices of targets and band pass(es) can reduce degeneracies from reddening and period distribution differences, and comparison of the Cepheid distance scale with other methods for galaxies with a wide range of environmental conditions is instructive (Mould \& Sakai 2008, 2009a.b; Hislop et al. 2011). Treating this controversial topic (Sakai et al. 2004; Romaniello et al. 2009) in detail is beyond the scope of this review. 
Storm et al. (2011a, b) apply a newly revised calibration of the infrared surface brightness technique to $41 \mathrm{MC}$ stars (36 LMC, 5 SMC) and derive a distance modulus for the LMC of $18.46 \pm 0.04$ (statistical) where the zero-point is anchored to the Cepheids with HST parallaxes and thus carries its own uncertainty of another $\sim 5 \%$, and where the projection $(p)$ factor that converts observed radial velocities to pulsation velocities is constrained by being forced to show no variation in LMC distance as a function of pulsation period. In particular, the $K$-band PL relation is found to have very little dependence on metallicity, in addition to the well-known advantages of lack of reddening sensitivity and less intrinsic scatter than PL relations at shorter wavelengths. This result is consistent with that found by Freedman \& Madore (2009). Freedman \& Madore (2010) provide two recent analyses of LMC Cepheid data, each referenced to the Benedict et al. (2007) zero-point. The first is a multi-wavelength solution based on the apparent BVIJHK Cepheid distance moduli, fitting to the Cardelli et al. (1989) extinction curve, to find that the data are well-fit by an LMC distance modulus of $18.40 \pm 0.01 \mathrm{mag}$ and $E(B-V)=0.10 \mathrm{mag}$. The second method involves fitting the LMC Cepheids to the Galactic Cepheids with parallaxes in the reddening-free Wesenheit $W(V, I) \mathrm{PL}$ relation. With no correction for metallicity the LMC modulus so derived is $18.44 \pm 0.03 \mathrm{mag}$, with a correction corresponding to 0.25 $\mathrm{mag} / \mathrm{dex}$ (Sakai et al. 2004) the LMC modulus would decrease to 18.39.

We conclude that almost all the recent work, when zero-pointed from the Benedict et al. (2007) Galactic calibration, give LMC distance moduli in the range $18.40-18.50$, with mean about 18.45 mag. Effects arising from the difference in metallicity between the the LMC and the Galaxy are the present major source of systematic error, although recent evidence hints at the effect of metallicity on Cepheid absolute magnitudes being very small, particularly in $K$ (Storm et al. 2011a) and $W(V, I)$ (Majaess et al. 2011) band passes. Additionally, both these band passes are relatively insensitive to the effects of reddening. 


\section{Red Variables}

Miras are pulsating variable stars on the Asymptotic Giant Branch (AGB) stage of their evolution, with progenitor masses in the range $\sim 0.8-8 M_{\odot}$. Those most useful for distance scale work have periods in the range $200-400$ days where tight PL relations are found, particularly in the $K$ band e.g. Whitelock et al. (2008). Data from the rich MC samples in the newer surveys such as the OGLE III catalog (Soszyński et al. 2009), and cross identifying the stars in various IR catalogs show well-delineated PL relations. Careful comparisons between measurements in the different band passes appears to allow separation of stars with different surface compositions (e.g. C stars), different evolutionary states (e.g. supergiants with longer periods) and different pulsation modes. The effects of circumstellar material on the band passes (Ita \& Matsunaga 2011) can be significant. The large samples of stars also help to smooth out the "noise" of individual stars, which typically display 0.1 mag of secular variation in the $K$ band (Whitelock et al. 2008).

The analysis of the Hipparcos parallax data by Whitelock \& Feast (2000) was revised and extended by Whitelock et al. (2008) who also add $5 \mathrm{OH}$ masers with parallaxes from the ESO VLTI, and 11 Miras in globular clusters where the distance zero-point is based on Hipparcos parallaxes for subdwarfs (Carreta et al. 2000). The corresponding infrared photometry is almost exclusively from the SAAO. They divide their Hipparcos sample into carbon-rich and oxygen-rich samples, and then subdivide these samples in various ways, finding that carbon-rich stars give a similar zero-point but with larger uncertainty, and no significant effect with metallicity is found. Assuming the slope for the $\mathrm{PL}(K)$ relation as found in the LMC, their preferred solution for the O-rich galactic Miras is

$$
M_{K}=-3.51 \pm 0.20(\log P-2.38)-7.25 \pm 0.07
$$

and thus, taking in quadrature their error of $\pm 0.06 \mathrm{mag}$ for the zero-point error of 
their fit to the LMC Miras, a Mira distance modulus for the LMC of $18.49 \pm 0.09$ mag is obtained.

Tabur et al. (2010) used a complementary approach in their analysis of $K$-band light curves for 247 southern, semi-regular red variables with Hipparcos parallaxes, allocating the stars to the several sequences in the $\log P-M_{K}$ diagram (Wood 2000; Soszyński et al. 2004) and comparing to stars in the MC. They show the various P-L zero-points have a negligible metallicity dependence, and find an LMC modulus of $18.54 \pm 0.03$ (internal), plus the modulus difference between the LMC and SMC is $0.41 \pm 0.02 \mathrm{mag}$.

We conclude that the results from the red variables give an LMC distance modulus of close to 18.51 mag.

\section{RR Lyraes}

RR Lyraes are low mass $\left(\sim 0.6-0.8 M_{\odot}\right)$ core-helium burning stars that lie within the pulsational instability strip in the horizontal branch region of the Hertzsprung-Russell (HR) diagram. In the $V$ band the absolute magnitudes exhibit a dependence on metallicity that appears from both observations and theory to be linear over a wide range (at least $-2.5<[\mathrm{Fe} / \mathrm{H}]<-1.0$ ) and universal (Bono et al. 2008; Clementini 2008), with Gratton et al. (2004) finding by combining their spectroscopy of $\sim 100$ field RR Lyraes from the photometric study by Clementini et al. (2003) that

$$
M_{V}=(0.214 \pm 0.047)([F e / H]+1.5)+\text { constant } .
$$

However, this simple relation may not be valid in some environments, for instance Caputo (2011) cautions that the the luminosity on the horizontal branch is very dependent on the helium abundance $Y$, with $\Delta M_{V} / \Delta Y \sim-4.0,16$ times the effect of metallicity 
$Z$. Additionally, there is some evidence e.g. Di Criscienzo et al. (2004) that at the higher metallicities the simple relationship with $M_{V}$ becomes non-linear, while evolutionary effects can intrude when comparing RR Lyraes between globular clusters with differing distributions of horizontal branch stars. In the near infrared (NIR) observational data are well fit by a period-luminosity-metallicity (e.g. $P-L_{K}-Z$ ) relation, with the advantages and disadvantages of this method very clearly summarized by Coppola et al. (2011).

The recently released OGLE-III catalog of 24906 RR Lyraes stars in the LMC is an enormous increase in the numbers known and apart from the specific use as distance indicators the catalog is invaluable for studies of the LMC structure (Pejcha \& Stanek 2009). But even for earlier surveys the number of stars are substantial, e.g. the OGLE-II catalog contains over 500 RR Lyrae stars for the SMC (Soszyński et al. 2002). There are also a few old clusters in the LMC, and one in the SMC (NGC 121) that contain RR Lyraes, see e.g. Nemec et al. (2009).

The zero-point calibration rests on the parallaxes for five RR Lyrae stars, a calibration via globular clusters whose distances are determined via absolute magnitudes for subdwarfs as measured by Hipparcos, and from various theoretical and empirical relations. Catelan \& Cortes (2008) form a weighted mean of the three parallax measurements available (HST, Hipparcos, Ground) for RR Lyrae itself to obtain a final value of $\pi_{a b s}=3.78 \pm 0.19$ mas, corresponding to a distance modulus of $7.11 \pm 0.11 \mathrm{mag}$. By means of theoretical models relating absolute magnitudes for RR Lyrae stars to their periods and the Strömgren pseudo-color $c_{0}$ together with their calculated reddening and intensity-mean $V$ magnitude $(\langle V\rangle)$ they derive an absolute magnitude for RR Lyrae of $<M_{V}>=0.600 \pm 0.126$. Such careful analysis is essential when the calibration rests on a single star; the situation has improved very recently with the publication of parallaxes for five RR Lyrae stars (including an improved parallax for RR Lyrae) by Benedict et al. 
(2011), received as a preprint as this review was in preparation.

Distances to globular clusters can at present be most directly determined via fitting local subdwarfs whose distances have been determined by Hipparcos to the unevolved main sequence of the GC in the CMD (Carreta et al. 2000). Such calibrations require the cluster reddening and metallicity as input, and for well-studied globular clusters the distances so-obtained agree within the errors with other methods e.g. see Coppola et al. (2011) for M5. This results will soon be greatly strengthened by improved HST FGS parallaxes for more subdwarfs (Chabover et al. 2011). The NIR PL relations derived theoretically by Bono et al. (2003) and Catelan et al. (2004) also agree rather well with those using either of the above empirical methods.

There are a number of recent independent studies measuring RR Lyraes in the NIR in the LMC for both field and clusters (Borissova et al. 2004; Dall'Ora et al. 2004; Szewczyk et al. 2008), and the authors variously choose to use empirical or theoretical derivations for the zero-point. In all cases the error on the resulting distance modulus is $\sim 0.1-0.15 \mathrm{mag}$, and the range $18.48-18.58 \mathrm{mag}$. However, with the new parallaxes from Benedict et al. (2011) it is clear that using their absolute magnitude scale is the most robust way to establish the Population II distance scale at present; we quote their results for the LMC distance taking the mean of their two methods for bias corrections, Lutz-Kelker-Hanson (LKK) (Hanson 1979) and reduced parallaxes (RP), (Feast 2002), however the difference in LMC modulus between these is small, and they find that an RR Lyrae star with $[\mathrm{Fe} / \mathrm{H}]=-1.5$ has $M_{V}=0.45 \pm 0,05(\mathrm{LKK})$ or $M_{V}=0.46 \pm 0.03$ (RP). This is substantially brighter than the derived value of $M_{V}=0.66 \pm 0.14$ at $[F e / H]=-1.48 \pm 0.07$ (Catelan \& Cortes 2008) based on RR Lyrae alone, but with much smaller error. The results given below would indicate the analyses of $K$ band data based on RR Lyrae need to have their derived LMC modulus adjusted by only a small amount, 
$\sim 0.02$ mag when the new calibration is applied. The reasons for the smaller adjustment in the IR will be of interest to investigate.

The distance to the LMC from analyses of field RR Lyraes observed in optical band passes depends on the treatment of the reddening; this is not only a problem just for the RR Lyraes of course and is probably the strongest driver for moving to the infrared when at all possible. Also, three of the five galactic RR Lyrae stars with HST parallaxes have $E(B-V) \geq 0.1 \mathrm{mag}$, so assuming the $K$ magnitudes for the parallax stars are accurate (see Feast et al. (2008) for a description of how the $K$ band magnitudes were obtained), then the zero-point is potentially more robust in the infrared as well. Thus in the optical, the primary difference between distances derived from the data of Gratton et al. (2004) and Soszyński et al. (2003) is due to the different reddening used in the two studies, with Benedict et al. (2011) finding an LMC modulus of $18.61 \pm 0.05$ for the Gratton et al. (2004) data and $18.46 \pm 0.06$ for the Soszyński et al. (2003) data. In the infrared, they quote $18.55 \pm 0.05$ for the $\mathrm{K}$ band data analyzed by Borissova et al. (2009) and $18.50 \pm 0.03$ for the RR Lyraes in the Reticulum cluster measured by Dall'Ora et al. (2004).

For the SMC, a recent Fourier analysis of the light curves for 335 RRab and 17 RRc selected from an OGLE-II sample of 536 single-mode RR Lyraes by Deb \& Singh (2010) yields a distance modulus of $18.83 \pm 0.01 \mathrm{mag}$ (internal) using intensity-weighted mean magnitudes and an absolute magnitude calibration from Kovács \& Jursik (1996). It would be interesting to compare this result with that obtained with a more modern RR Lyrae zero-point calibration (some 0,2 mag brighter) and re-examination of the reddening corrections, Kapakos et al. (2011) similarly analyze 100 carefully selected RR Lyraes from the OGLE-II and OGLE-III catalogs, and calculate an SMC modulus of $18.90 \pm 0.18$ referenced to an LMC modulus of $18.52 \pm 0,06$. The RR Lyraes from the OGLE catalogs are clearly a very rich resource for delineating the structure of the MC as exhibited by its 
oldest populations, in addition to mean distances to the galaxies themselves.

We conclude that the RR Lyrae results for the LMC distance tend in the mean to give a result near $18.53 \mathrm{mag}$, with more dispersion than is desirable in the optical analyses, due to differing treatments of reddening. Further analysis of the OGLE data together with the new Galactic calibration, should allow the situation to be dramatically improved.

\section{Red Clump Stars}

Red clump stars are common in the solar vicinity and many have accurate Hipparcos parallaxes allowing an accurate mean absolute magnitude for the solar sample to be derived (Alves 2000; Groenwegen 2008). Several authors e.g. Percival \& Salaris (2003) and references therein have shown that substantial corrections are needed for age and metallicity, and that these are very dependent on the band pass, for instance if the stars are around 2 Gyr old then the $K$ band is excellent with no metallicity correction needed, whereas if the stars are very old the $I$ band is better, and generally the $V$ band should be avoided (Salaris et al. 2003). This conclusion was also reached empirically by Pietrzyński et al. (2003). The theoretically derived population corrections and in particular the $\mathrm{K}$ band mean absolute magnitude found by Salaris et al. (2003) are in excellent agreement with the revised Hipparcos parallaxes for red clump stars (Groenwegen 2008). Thus, Salaris et al. (2003) find a distance modulus for the LMC of $18.47 \pm 0.01$ (random) \pm 0.06 (systematic) using the LMC photometry by Pietrzyński et al. (2003) who from their own analysis and the Alves (2000) calibration find an LMC modulus of $18.50 \pm 0.01$ (internal), while analysis of an independent data set by Koerwer (2009) finds $18.54 \pm 0.06$. However, measuring red clump $K$ magnitudes for 17 intermediate age LMC clusters, Grocholski et al. (2007) find a rather shorter mean distance, $18.40 \pm 0.04$ (random) \pm 0.08 (systematic). 
In summary, there is now deep understanding on how to determine distances from red clump stars, and providing the caveats are respected the method can be very reliable as the stars are relatively common. The results cluster around an LMC modulus of 18.48.

\section{Eclipsing Binaries}

Detached binaries that are spectroscopically double-lined and also photometrically eclipsing allow the measurement of the fundamental stellar parameters for the two companions, with the distance able to be determined with the addition of the absolute surface brightness, first pointed out by Lacey (1977). The absolute surface brightness can be found empirically from the surface brightness - color relation which is well established for stars of spectral type later than A5 from interferometrically-measured stellar angular diameters. For early type stars the process is less empirical and at present relies on accurately determining the effective temperatures e.g. Bonanos et al. (2011). But in principle, the use of detached double-lined eclipsing binaries for determining distances with a minimum of assumptions (Andersen 1991; Torres et al. 2010) has the potential to allow one-step distances to several nearby Local Group galaxies (Paczynski 1996; Hilditch 2006). Until very recently this type of analysis in the LMC has been restricted to a few early type systems (Guinan et al. 1998; Ribas et al. 2002; Fitzpatrick et al. 2002, 2003), and to 21 detached systems in the SMC (Hilditch et al. 2005). The first LMC results tended to give rather short distances, $18.30 \pm 0.07$ (Guinan et al. 1998), $18.38 \pm 0.08$ (Ribas et al. 2002), $18.53 \pm 0.05$ (Fitzpatrick et al. 2002), $18.18 \pm 0.08$ (Fitzpatrick et al. 2003). The latter system has low (galactic only) reddening which would support it being foreground to the main body of the LMC, as suggested by Fitzpatrick et al. (2003). The faintness of late type systems at the distance of the MC makes their study difficult, however OGLE III discoveries of $\mathrm{G}$ giant systems will enhance the sample of LMC detached eclipsing 
binaries considerably and allow a more direct derivation of distance than with the early type systems, with smaller expected systematic uncertainty. The first of these new analyses,

for a system consisting of two G4III stars, has been published by Pietrzyński et al (2009). Their derived value for the distance of this system is $18.50 \pm 0.06 \mathrm{mag}$, using the $V-K$ surface brightness - color relation of di Benedetto (2005). Pietrzyński et al. (2010) point out that with the discovery of a few dozen such systems in the MC we will be able to determine the distances to both galaxies to $1-2 \%$ accuracy from this method alone.

In summary, unlike the other LMC distance indicators discussed above that due to their copious numbers can have the statistical errors in their mean apparent distance reduced to small levels, there are at present only six detached double-lined eclipsing binaries in the LMC with published distances. Three of these cluster closely around a modulus of $18.52 \mathrm{mag}$, the other three have much shorter moduli. Setting aside the very discrepant value for HV5936 (18.18 mag), the remaining five systems provide a mean LMC modulus of 18.45 mag.

\section{Conclusions}

The distance indicators discussed above show remarkable agreement. The mean of the mean distances stated above at the end of each section for the five distance indicators is $18.48 \mathrm{mag}$, and the range is $\pm 0.05 \mathrm{mag}$. With most of the measurements discussed quoting internal errors typically $0.01-0.03 \mathrm{mag}$, and with the inclusion of external (calibration) errors typically $0.04-0.09 \mathrm{mag}$, adopting the mean of the means and the range as our present best estimate for the distance of the LMC and its error seems justifiable. The Cepheid result (18.45 mag) is uncorrected for the metallicity difference between the Galaxy and the LMC; a corollary is that such a correction cannot be very large. 
An accurate distance scale is a prerequisite for studying many of the most profound question in astronomy, and the MC. particularly the LMC, continue to play a pivotal role in improving and understanding a variety of distance indicators. Major recent surveys of the MC have provided enormous increases in the numbers of such stars, helping to improve our understanding of their properties and allowing the preparation of unbiased samples for comparison in detail with stars in our own Galaxy and elsewhere. The sample sizes are such that calibration and cross-calibration effects are becoming dominant, however the demands of the target science are such that these effects need to be understood at the $\sim 0.02 \mathrm{mag}$ level, and preferably at least a factor of two better. The several standard systems in the near IR require very careful transformation work, and similar procedures will be needed in the optical, where the tendency for the new wide field instruments becoming available in the south on large telescopes to survey in the filters approximating the SDSS system will complicate comparisons with legacy data obtained in the Johnson-Cousins system.

The zero-point calibrations have improved for both Cepheids and RR Lyraes. Hipparcos first provided parallaxes for Cepheids and red clump stars, while HST FGS has provided high quality measurements for a few Cepheids and RR Lyraes, and should soon also further strengthen the Population II scale with good parallaxes for a few subdwarfs. GAIA will make dramatic improvements with factors of at least a hundred increase in both sample sizes and astrometric accuracy. Eclipsing binaries also provide a very fundamental way to determine the distances to the $\mathrm{MC}$, and here the discovery of G III examples, allowing a more straightforward analysis, is a major advance. Improved calibrations and analysis techniques for the early type stars are also providing more reliable results for these stars.

Finally, intense efforts on understanding the evolutionary properties of post main sequence turnoff stars, and in particular the properties of pulsating variables, has placed analysis of the observational results on a very sound theoretical footing. Although there 
are still important effects that elude us, such as the luminosity dependence of Cepheids on metallicity, and determining how pervasive is the need to vary helium content, there has been substantial progress, with the red clump stars now a well-understood distance indicator being a prime example of this. However, we are not home yet. The desire to know the Hubble Constant to $\sim 1 \%$ places great demands on both observations and theory, it will require extraordinary care to improve the accuracy of the present result by another factor of two or three. 


\section{REFERENCES}

Alves, D.R. 2000, ApJ, 539, 732

Alves, D.R. 2005, Highlights in Astronomy, IAU, O. Engvold, San Francisco, CA: Astronomical Society of the Pacific, Vol. 13, 1448

Andersen, J. 1991, A\&A Rev., 3, 91

Benedict, G.F., McArthur, B.E., Fredrick, L.W., et al. 2002, AJ, 124, 1695

Benedict, G.F., McArthur, B.E., Feast, M.W., et al. 2007, AJ, 133, 1810

Benedict, G. F., McArthur, B. E., Feast, M.W., et al. 2011, AJ, 142, 187

Besla, G., Kallivayalil, N., Hermquiat, L., et al. 2010, ApJ, 721, L97

Bonanos, A.Z., Castro, N., Macri, L.M., Kudritzki, R-P. 2011, ApJ, 729, L9

Bono, G., Caputo, F., Castellani, V., et al. 2003, MNRAS, 344, 1097

Bono, G., Caputo, F., Fiorentino, G, Marconi, M., \& Musella, I. 2008, ApJ, 684, 102

Borissova, J., Minniti, D., Rejkuba, M., et al. 2004, A\&A, 423, 97

Borissova, J., Rejkuba, M., Minniti, D., Catelan, M., \& Ivanov, V. D. 2009, A\&A, 502, 505

Caldwell, J.A.R., \& Coulson, I.M. 1986, MNRAS, 218, 223

Caputo, F., 2011, this meeting

Cardelli, J.A., Clayton, G.G., \& Mathis, J.S. 1989, AJ, 96, 695

Carretta, E., Gratton, R.G., Clementini, G., \& Fusi Pecci, F. 2000, ApJ, 533, 215

Catelan, M., Pritzl, B.J., \& Smith, H.A. 2004, ApJS, 154, 633 
Catelan, M., \& Cortes, C. 2008, ApJ, 676, L135

Chaboyer, B. C., Benedict, G. F., McArthur, B. E., et al. 2011, Bulletin of the American Astronomical Society, 43, \#242.25

Clementini, G., Gratton, R.G., Bragaglia, A.,et al. 2003, AJ, 125, 1309

Clementini, G. 2008, IAU Symposium 256, J. Th. van Loon \& J.M. Oliveira, Cambridge: CUP, 373

Coppola G., Dall'Ora, M., Ripepi, V., et al. 2011, MNRAS, 416, 1056

Dall'Ora, M., Storm, J., Bono, G., et al. 2004, ApJ, 610, 269

Deb, S., \& Singh, H.P. 2010, MNRAS, 402, 691

di Benedetto, G.P. 2005, MNRAS, 357, 174

Di Criscienzo, M., Marconi, M., \& Caputo, F. 2004, ApJ, 612, 1092

Feast, M.W. 2002, MNRAS, 337, 1035

Feast, M.W., Laney, C.D., Kinman, T.D., Van Leeuwen, F., \& Whitelock, P.A. 2008, MNRAS, 386, 2115

Feast, M.W. \& Catchpole, R.F. 1997, MNRAS, 286, L1

Feast, M.W. \& Walker, A.R. 1987, ARA\&A, 25, 345

Fitzpatrick, E.F., Ribas, I., Guinan, E.F., et al. 2002, ApJ, 564, 26-

Fitzpatrick, E.F., Ribas, I., Guinan, E.F., Maloney, F.P., Claret, A. 2003, ApJ, 587, 685

Fouqué, P., Arriagada, P., Storm, J., et al. 2007, A\&A, 476, 73

Freedman, W.L., \& Madore, B.F. 2009, ApJ, 696, 1498 
Freedman, W.L. \& Madore, B.F. 2010, ARA\&A, 48, 673

Freedman, W.L. \& Madore, B.F. 2011, ApJ, 734, 46

Gieren, W., Storm, J., Barnes T.G., et al. 2005, ApJ, 627, 224

Gratton, R.G., Bragaglia, A., Clementini, G., et al. 2004, A\&A, 421, 937

Grocholski, A., Sarajedini, A., Olsen, K.A.G., Tiede, G.P., \& Mancone, C.L. 2007, AJ, 134, 680

Groenwegen, M. 2008, A\&A, 488, 935

Guinan, E.F., Fitzpatrick, E.L., Dewarf, L.E., et al.1998, ApJ, 509, L21

Hanson, R.P. 1979, MNRAS, 186, 875

Hilditch, R.W., Howarth, I.D., \& Harries, T.J. 2005, MNRAS, 357, 304

Hilditch, R.W. 2006, Ap\&SS, 304, 203

Hislop. L., et al. 2011, ApJ, 733, 79

Ita, Y. \& Matsunaga, N. 2011, MNRAS, 412, 2345

Kapakos, E., Hatzidimitriou, D., \& Soszyński, I. 2011, MNRAS, 415, 1366

Koewer, J.F. 2009, AJ, 138,1

Komatsu,E., Smith, K.M., Dunkley, J., et al. 2011, ApJS, 192, 18

Kovács, G. \& Jursik, J. 1996, ApJ, 466, 47

Kudritzki, R-P., Urbaneja, M.A., Bresolin, F., et al. 2008, ApJ, 681, 269

Lacey, C. 1977, ApJ, 213, 458 
Macri, L.M., Stanek, K.Z., Bersier, D., Greenhill, L.J., \& Reid, M.J. 2006, ApJ, 652, 1133

Madore, B.F. \& Freedman, W.L. 2009, ApJ, 696, 1498

Majaess, D., Turner, D. \& Gieren, W. 2011, ApJ, 741. L36

Mould, J., \& Sakai, S. 2008, ApJ, 686, 75

Mould, J., \& Sakai, S. 2009a, ApJ, 694, 1331

Mould, J., \& Sakai, S. 2009b, ApJ, 697, 996

Nemec, J.N., Walker, A.R., \& Jeon, Y-B. 2009, AJ, 138, 1310

Nidever, D.L., Majewski, S.R., Butler Burton, W., \& Nigra, L. 2010, ApJ, 723, 1618

Nikolaev, S., Drake, A.J., Keller, S,C., et al. 2004, ApJ, 601,260

Olsen, K.A.G., Zaritsky, D, Blum, R.D., Boyer, M.L., \& Gordon, K.D. 2011, ApJ, 737, 29

Paczynski, B. 1996, Extragalactic Distance Scale STScI Symp., arXiv:astro-ph/9608094

Percival, S., \& Salaris, M. 2003, MNRAS, 343, 539

Pejcha, O., \& Stanek, K.Z. 2009, ApJ, 704, 1730

Pietryński, G., Gieren, W, \& Udalski, A 2003, AJ, 125, 2494

Pietryński, G., Thompson, I.B., Graczyk, D., et al. 2009, ApJ, 697, 862

Pietryński, G., Thompson, I., Graczyk, D., Gieren, W., \& Minniti, D. 2010, ASP Conf. Ser., 435,13

Ribas, I., Fitzpatrick, E.L., Maloney, F.P., Guinan, E.F., Udalski, A. 2002, ApJ, 574, 771

Riess, A.G., et al., 2011, ApJ, 730, 119 
Romaniello, M., Primas, F., Mottini, M., et al. 2009, AIPC, 1170, 99

Saha, A., Olszewski, E.W., Brondel, B., et al. 2010, AJ, 140, 1719

Sakai, S., Ferrarese, L., Kennicut, R.C., \& Saha, A. 2004, ApJ, 608, 42

Salaris, M., Percival, S., \& Girardi, L. 2003, 345, 1030

Schaefer, B.E. 2008, AJ, 1354,112

Scowcroft, V., Bersier, D., Mould, J.R., \& Wood, P.R. 2009, MNRAS, 396, 1287

Sollima, A., Cacciari, C., \& Valenti, E. 2006, MNRAS, 372, 1675

Soszyński, I., Udalski, A., Szymański, M., et al. 2002, Acta Astron., 52, 369

Soszyński, I., Udalski, A., Szymański, M., et al. 2003, Acta Astron., 59, 1

Soszyński, I., Udalski, A., Kubiak, M., et al. 2004, Acta Astron., 54, 129

Soszyński, I., Udalski, A., Szymański, M., et al. 2009, A\&A, 59, 239

Storm, J., Gieren, W., Fouqué,P., 2011a, A\&A, 534, 94

Storm, J., Gieren, W., Fouqué,P., 2011b, A\&A, 534, 95

Szewczyk, O., Pietrzyński, G., Gieren, W., et al. 2008, AJ, 136, 272

Tabur, V., Bedding, T. R., Kiss, L. L., et al. 2010, MNRAS, 409, 777

Tammann, G.A., Sandage, A., \& Reindl, B. 2008, ApJ, 679, 52

Torres, G, Andersen, J., \& Giménez, A. 2010, A\&A Rev., 18, 67

Turner, D.G. 2010, Ap\&SS, 326, 219

Turner, D.G. \& Burke, J.F. 2002, AJ, 124, 2931 
van Leeuwen, F., Feast, M.W., Whitelock, P.A., \& Laney, C.D. 2007, MNRAS, 379, 723

Walker, A.R. 2003, Stellar Candles for the Extragalactic Distance Scale, Lecture Notes in Physics, D. Alloin \& W. Gieren, 635, 265

Whitelock, P.A. \& Feast, M.W. 2000, MNRAS, 319, 759

Whitelock, P.A., Feast, M.W. \& van Leeuwen, F. 2008, MNRAS, 386, 313

Windmark, F., Lindgren, L., Hobbs, D. 2011, A\&A, 530, 76

Wood, P. R. 2000, PASA, 17, 18 